\author{
Crispin Jenkinson \\ Ray Fitzpatrick \\ Cathy Brennan \\ Mark Bromberg \\ Michael Swash
}

\section{Development and validation of a short measure of health status for individuals with amyotrophic lateral sclerosis/ motor neurone disease: the ALSAQ-40}

C. Jenkinson $(\bowtie) \cdot C$. Brennan Health Services Research Unit, Division of Public Health \& Primary Health Care, University of Oxford, Institute of Health Sciences, Headington, Oxford, OX3 7LF, UK e-mail: crispin.jenkinson@dphpc.ox.ac.uk

R. Fitzpatrick

Nuffield College, Oxford, OX1 1NF, UK

M. Bromberg

Department of Neurology,

Neuromuscular Program

and EMG Laboratory,

The University of Utah,

Salt Lake City, Utah 84132, USA

M. Swash

Department of Neurology,

St Bartholomew's

and the Royal London Hospital School

of Medicine and Dentistry,

(Queen Mary and Westfield College,

University of London),

Whitechapel, London, E1 1BB, UK

\begin{abstract}
In recent years there has been an increased appreciation of the importance of measuring health status from the patient's point of view, but until now no attempt has been made to develop an amyotrophic lateral sclerosis (ALS)-specific health status measure. The development of such an instrument is especially relevant now with the introduction of drugs that prolong life in ALS but limited data is available on the impact such treatments have on quality of life. This paper reports on the development of an ALS-specific measure, the forty item ALS assessment questionnaire (ALSAQ-40). The development of the ALSAQ-40 followed three main stages. Stage 1 consisted of in-depth, semi-structured exploratory interviews conducted on a sample of 18 patients to identify areas of salience and concern to patients with ALS. These interviews generated 78 candidate questions. In stage 2 , the 78 -item questionnaire
\end{abstract}

was used in a postal survey to identify appropriate rephrasing/shortening and to determine the acceptability of the measure. In addition, this exercise helped identify sub-scales of the instrument addressing different dimensions of ALS. Finally in stage 3 the data collected in stage 2 was analysed to areas measured by the instrument and to remove redundant questions. The resulting measure contains forty questions measuring five areas of health status: Eating and Drinking, Communication, ADL/ independence, Physical mobility, Emotional Functioning. The measure has high face, internal and construct validity and is likely to prove a useful measure in the evaluation of treatment regimes for ALS/MND.

Key words Amyotrophic lateral sclerosis - Quality of life . Disease-specific measure . ALSAQ-40

\section{Introduction}

The motor neurone diseases (MND) are progressive, fatal, neurodegenerative disorders characterised by progressive loss of motor neurones. By far the most common form of MND is amyotrophic lateral sclerosis (ALS) and these two terms are sometimes used synonymously. The disease occurs world-wide with an incidence of approximately $1-1.5$ per 100,000 population and a prevalence of $4-6$ per 100,000 [5]. It is more common in men than women (in a ratio of approximately $3: 2$ ) and the incidence of the disease rises with age, with onset typically occurring in mid to late $50 \mathrm{~s}$. The average survival time from diagnosis is approximately 2-3 years [2]. The course of the disease is usually relentlessly progressive, although it can be protracted, especially amongst those contracting it at younger ages [12]. The disease is often associated with psychological reactions of depression, feelings of loss of control, fear, frustration, isolation and anxiety [7]. Although there is currently no cure for ALS, evidence from clinical trials shows that a glutamate antagonist (riluzole) extends sur- 
vival and slows aspects of the rate of disease progression [1]. Other drugs are also in development.

A number of instruments exist to measure health status and health-related quality of life. For example, the SF-36 health survey questionnaire is a short (36 item) general measure of health status that can be used to assess functioning and well being in any patient group. This measure has been used in ALS/MND [10]. Similarly, the 136-item Sickness Impact Profile (SIP) has been recommended as an appropriate generic measure in this patient group. Despite its length, shortened versions could reduce its precision and sensitivity to change [4]. However, the application of such measures in ALS/MND has been relatively rare and, furthermore, there exists no disease-specific measure for this unique patient group. This is striking given that treatment for ALS/MND is not curative and the purpose of interventions is to improve quality of life and increase survival. Consequently, the purpose of this paper is to present the results of a study to develop and validate an ALS/MND specific measure, namely the 40-item ALS Assessment Questionnaire (ALSAQ-40). This instrument was developed and tested by three stages of work.

\section{Stage 1 - Item generation}

Exploratory in-depth interviews were carried out in patients with ALS in order to generate a large number of candidate questionnaire items. A questionnaire was developed which contained 78 items.

Stage 2 - Item reduction and scale generation

A postal survey was conducted using the 78 -item questionnaire generated at stage 1 in order to determine the acceptability of the measure. Furthermore, this phase enabled the development of a shorter, and hence more practical instrument with a smaller number of items and the identification of the sub-scales of the instrument which address different dimensions of ALS.

\section{Stage 3 - Testing construct validity}

Two small-scale follow-up surveys were conducted to test the validity of the questionnaire in comparison with an established general measure of self-reported health status, the SF-36 [16], and a clinical measure of outcome, the ALS Functional Rating Scale (ALS-FRS) [13]. It was hypothesised that scales with similar content would be significantly correlated, although given the highly specific nature of the ALSAQ-40, and the fact it is completed by the patient, such correlations would not be perfect.

\section{Methods}

Stage 1 - item generation

In-depth semi-structured interviews with 18 patients presenting with ALS/MND were tape-recorded. The point at which no new significant themes appeared to emerge from the interviews determined the sample size for this stage of the study. Patients of different ages and presenting at different phases of ALS/MND were asked to describe the areas of their lives that had been influenced by their ALS/MND, and a list of aspects of life adversely affected by the disease was extracted from the transcribed interviews. Four researchers went on to independently devised questionnaire items from this list, which were then discussed, scrutinised for repetition and ambiguity, and a final set of items agreed. This lead to a final pool of 78 items, which asked about the influence of ALS on a specific area of life over the past two weeks. Respondents were requested to select one of the following answers: Never (0); Occasionally (1); Rarely (2); Often (3); Always / cannot do at all (4) for each question. The face validity of the questionnaire was also assessed at this stage by two patients, a regional care advisor and two neurologists. Consequently 75 copies of the instrument were sent to patients with MND, with a request to complete the questionnaire and indicate appropriate alterations. A telephone number was also provided so that patients could call with comments. Few comments were made about the measure and no single issue was consistently raised. Consequently, it was decided to make no alterations to the measure.

Stage 2 - Item reduction and scale generation

The 78-item questionnaire was posted to a sample of individuals with ALS. Care advisors for regions of the Motor Neurone Disease Association Society in England, Wales and Northern Ireland were approached to help recruitment. A small number of patients $(n=$ 25) were not contacted in this way but volunteered to take part in the study in response to an article in 'Thumbprint', the UK MND Association newsletter. Overall, a total of 208 individuals received the questionnaire; 173 patients completed and returned the questionnaire (29 non-responders, 6 returned blank questionnaires). Thus a response rate of $83.2 \%(173 / 208)$ was achieved. The mean age of the sample was 62.6 years (standard deviation [SD] 12.5; $\min =31$ years, $\max =92$ years, $n=168)$, with $66(38.2 \%)$ being female and $104(60.1 \%)$ male. The mean period since diagnosis was 37.9 months (SD 41.1; $\min =2$ months; $\max =211, n=165$ ).

\section{Stage 3 - Testing construct validity}

The aim of this part of the study was to assess the construct validity of the questionnaire by analysing the first postal survey (reported below in Results). The disease-specific measure was administered with the SF-36 in a small-scale postal survey. It was sent to 44 patients of whom carers indicated that 4 had died. Completed questionnaires were returned by $35(87.5 \%)$ patients, 21 $(60 \%)$ were male and 14 female $(40 \%)$. The average age of patients was 63.5 years $(\mathrm{SD}=11.4, \min =36$ years, $\max =79$ years, $n=34)$ and the mean time since diagnosis was 41.5 months (SD $=$ 39.2 ; $\min =2$ months, $\max =136$ months, $n=34$ )

The acceptability of the questionnaire was also assessed in a small pilot study ( $n=17,10$ male: 7 female) undertaken in a neurology clinic in the USA. The average age of patients was 60.88 years $(\mathrm{SD}=8.64 ; \min =47$ years, $\max =78$ years, $n=17)$. Patients were also assessed by their clinician using the ALS-FRS. 
Table 1 ALSAQ-40 scale scores from the respondents of the first postal survey

\begin{tabular}{|c|c|c|c|}
\hline \multicolumn{4}{|l|}{ Scale $^{\mathrm{a}}$} \\
\hline \multicolumn{4}{|l|}{ Eating and Drinking } \\
\hline Mean & 39.40 & (\% scoring $\min )$ & $(25.4)$ \\
\hline Median & 33.33 & (\% scoring $\max )$ & $(4.7)$ \\
\hline (SD) & $(32.18)$ & (25th percentile) & $(0)$ \\
\hline (range of scores) & $(0-100)$ & (50th percentile) & (33.33) \\
\hline (n) & (169) & ( 75 th percentile) & $(58.22)$ \\
\hline \multicolumn{4}{|l|}{ Communication } \\
\hline Mean & 48.89 & (\% scoring $\min )$ & $(22.8)$ \\
\hline Median & 50.00 & $(\%$ scoring $\max )$ & $(2.4)$ \\
\hline (SD) & $(38.30)$ & (25th percentile) & $(3.57)$ \\
\hline (range of scores) & $(0-100)$ & (50th percentile) & $(50.0$ \\
\hline (n) & $(167)$ & ( 75 th percentile) & $(85.71)$ \\
\hline \multicolumn{4}{|l|}{ ADL/independence } \\
\hline Mean & 69.02 & (\% scoring $\min )$ & $(1.8)$ \\
\hline Median & 75.00 & $(\%$ scoring $\max )$ & (0) \\
\hline (SD) & $(28.20)$ & (25th percentile) & $(45.0)$ \\
\hline (range of scores) & $(0-100)$ & (50th percentile) & $(75.0)$ \\
\hline (n) & (166) & ( 75 th percentile) & $(95.0)$ \\
\hline \multicolumn{4}{|l|}{ Physical Mobility } \\
\hline Mean & 68.09 & (\% scoring $\min )$ & $(1.8)$ \\
\hline Median & 67.5 & (\% scoring $\max )$ & $(1.2)$ \\
\hline (SD) & $(28.71)$ & (25th percentile) & $(47.5)$ \\
\hline (range of scores) & $(0-100)$ & (50th percentile) & $(67.5)$ \\
\hline (n) & $(166)$ & (75th percentile) & $(97.5)$ \\
\hline \multicolumn{4}{|l|}{ Emotional functioning } \\
\hline Mean & 47.95 & (\% scoring $\min )$ & $(0.6)$ \\
\hline Median & 47.50 & (\% scoring $\max )$ & $(5.4)$ \\
\hline (SD) & (26.64) & (25th percentile) & $(25.0)$ \\
\hline (range of scores) & $(0-100)$ & (50th percentile) & $(45.0)$ \\
\hline (n) & (166) & ( 75 th percentile) & $(67.5)$ \\
\hline
\end{tabular}

${ }^{a}$ Each scale has a range from 0 (best possible health as measured by the scale) through to 100 (worst health as measured by the scale)

$\mathrm{SD}=$ Standard deviation

\section{Results}

Item reduction and scale generation

The data from the first postal survey (stage 2 in Methods) were factor analysed (varimax rotation) to determine the underlying dimensions of the 78-item questionnaire. Only factors which gained an eigenvalue in excess of 1 were initially retained. Items with a loading of $\leq 0.5$ for any of the factors were omitted. This produced 13 factors, 7 of which (accounting for $65.85 \%$ of variance) seemed both meaningful and interpretable. These 7 factors included 68 items loading above 0.5 on factors, which appeared to address the following aspects of health status: 1. talking and eating (16 items); 2. ADL/independence (16 items); 3. physical mobility (13 items); 4. emotional reactions (12 items); 5. fears about the future ( 3 items); 6 . financial limitations ( 2 items); 7. concerns about breathing ( 2 items).

It was assumed that items loading on each factor constituted a scale. Internal reliability was assessed using the Cronbach's alpha statistic on the items constituting each
Table 2 Corrected item to total correlations and internal reliability (Cronbach's alpha) of scales generated from the respondents with ALS/MND of the first postal survey

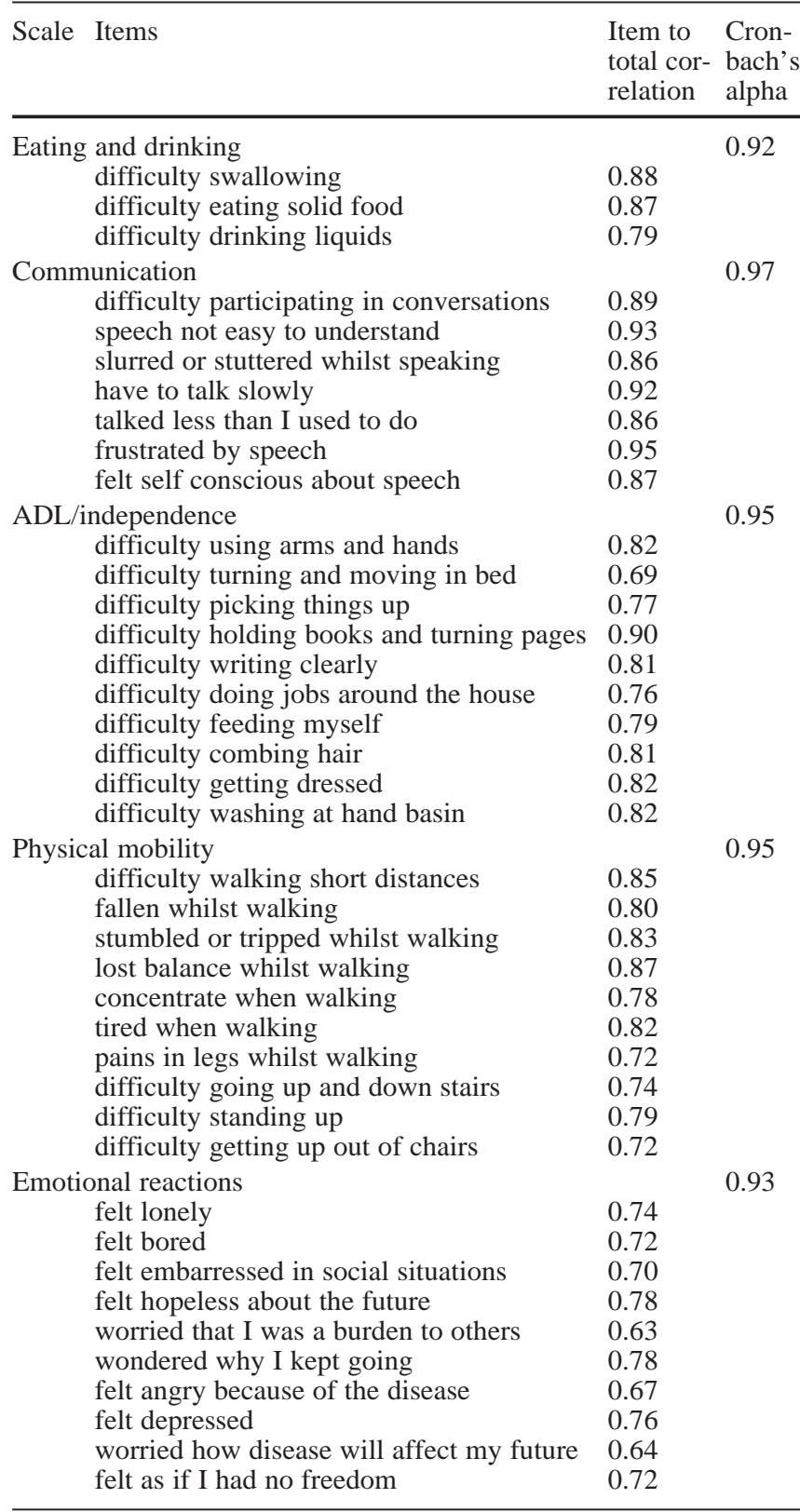

scale [3]. Consequently, items loading on factors 5, 6, and 7 were removed from the analysis because internal reliability was found to be low. Items were removed from each of the scales if they increased the alpha coefficient. In the case of factor 1 all items concerned with eating and swallowing were removed from the scale as this increased the internal reliability. At this stage it was decided to create a separate scale including the items addressing eating and swallowing items. A high internal reliability was gained and an eating scale developed. The remaining items on 
Table 3 Internal reliability of scales of the ALSAQ-40: the follow up survey of respondents with ALS/MND $(n=32)$ in the UK and in the US clinic dataset $(n=17)$

\begin{tabular}{lll}
\hline Scale & UK Alpha & US Alpha \\
\hline Eating and drinking & 0.94 & 0.94 \\
Communication & 0.98 & 0.97 \\
ADL/independence & 0.95 & 0.94 \\
Physical mobility & 0.94 & 0.96 \\
Emotional functioning & 0.93 & 0.91 \\
\hline
\end{tabular}

the original talking and eating scale were assessed for reliability, and consequently more items were removed, and the communication scale was developed. Although these two scales are strongly related (they indicate bulbar involvement) they are undeniably conceptually distinct. The remaining factors (ADL/independence, physical mobility, emotional reactions) were assessed for internal reliability and items removed until only those contributing maximally to the scale remained. These procedures resulted in a set of 40 questions incorporated in the following five scales:

- Eating and Drinking (3 items): addresses problems eating solid foods, swallowing and drinking liquids.

- Communication (7 items) addresses a variety of problems in communicating with others, for example difficulties with speech such as talking slowly, stuttering whilst speaking and feeling self-conscious about speech.

- Activities of daily living/independence (10 items) addresses a variety of limitations in ADL, for example, difficulties in washing/dressing oneself, doing tasks around the house, as well as difficulty writing.

- Mobility (10 items) addresses problems of mobility, for example, falling and difficulties in walking, standing up and going up and down the stairs.

- Emotional well-being (10 items) addresses various emotional problems, for example, feeling lonely, bored, depressed, feeling embarrassed in social situations and feeling worried about future disease progression.
Because the function of the ALSAQ-40 is to indicate the extent of ill health in each domain assessed, each scale was transformed to have a range from 0 (the best health status as measured on the questionnaire) through to 100 (the worst health status as measured on the questionnaire) with each scale being calculated as follows (Table 1):

$$
\text { Scale score }=\frac{\text { Total of raw scores of each scale item }}{\text { Maximum possible raw score of all }} \times 100
$$

The correlations of items with their scale totals and the internal consistency reliability of scales (i.e. the extent to which items in a scale tap a single underlying dimension) from the first survey were also determined (Table 2). Items were highly correlated with their own scale score (corrected to exclude the item to which it was being correlated). All the scales have very high internal consistency reliability by any standards, but are particularly high by the standard for group comparisons $[8,15]$.

Internal consistency reliability of the ALSAQ-40 was again assessed for the five dimensions in the small-scale follow-up study. Despite the relatively small sample size all dimensions showed very high internal consistency reliability (Table 3).

Construct validity (Spearman's non-parametric correlation coefficient [rho]) was assessed in two small-scale studies. In the first it was examined by means of correlations of scales for ALSAQ-40 with relevant scales for SF36. Mobility (ALSAQ-40) correlated with physical function $(\mathrm{SF}-36)(\mathrm{rho}=0.65, \mathrm{p}<0.001)$. Activities of daily living/independence (ALSAQ-40) correlated with both physical function $(\mathrm{SF}-36)(\mathrm{rho}=0.55, \mathrm{p}<0.001)$ and role limitations due to physical problems (SF-36) $(r h o=0.53$, $\mathrm{p}<0.001)$. Emotional well-being (ALSAQ-40) correlated with both mental health (SF-36) (rho $=0.64, \mathrm{p}<0.001)$, and role limitations due to mental problems (rho $=0.64$, $\mathrm{p}<0.001)$.

The acceptability and construct validity of the ALSAQ-40 was also assessed in a pilot study undertaken in a neurology clinic in the USA. Patients had little trouble completing the forms. Internal reliability of the measure was found to be comparable to that gained in the UK sam-
Table 4 Correlation coefficients (Spearman) between dimensions on the ALSAQ-40 and the ALS-FRS

$$
\begin{aligned}
& (n=15) . * * * \mathrm{p}<0.001 \\
& * * \mathrm{p}<0.01 ; * \mathrm{p}<0.05 \\
& \mathrm{NS}=\text { Not significant }
\end{aligned}
$$

\begin{tabular}{llllll}
\hline & $\begin{array}{l}\text { Eating \& } \\
\text { drinking }\end{array}$ & $\begin{array}{l}\text { Communi- } \\
\text { cation }\end{array}$ & $\begin{array}{l}\text { ADL/inde- } \\
\text { pendence }\end{array}$ & $\begin{array}{l}\text { Physical } \\
\text { mobility }\end{array}$ & $\begin{array}{l}\text { Emotional } \\
\text { reactions }\end{array}$ \\
\hline Speech & $0.54^{*}$ & $0.83^{* * *}$ & NS & NS & NS \\
Salivation & NS & $0.53^{*}$ & NS & NS & NS \\
Swallowing & $0.72^{* * *}$ & $0.92^{* * *}$ & NS & NS & NS \\
Handwriting & NS & NS & $0.57^{*}$ & NS & NS \\
Cutting food & NS & NS & $0.76^{* * *}$ & NS & NS \\
Dressing and Hygiene & NS & NS & $0.74^{* * *}$ & NS & NS \\
Turning in bed & NS & NS & $0.86^{* * *}$ & $0.80^{* * *}$ & $0.65^{* *}$ \\
Walking & $\mathrm{NS}$ & $\mathrm{NS}$ & $0.76^{* * *}$ & $0.89^{* * *}$ & $\mathrm{NS}$ \\
Climbing stairs & $\mathrm{NS}$ & $\mathrm{NS}$ & $0.69^{* *}$ & $0.95^{* * *}$ & $\mathrm{NS}$ \\
Breathing & $0.62^{* *}$ & $0.52^{*}$ & $\mathrm{NS}$ & $\mathrm{NS}$ & $\mathrm{NS}$ \\
\hline
\end{tabular}


ples (Table 3). High correlations were found between similar domains on a clinician completed form (ALS-FRS) and the ALSAQ-40 (Table 4). The only unexpected association was between emotional reactions (ALSAQ-40) and the turning in bed dimension (ALS-FRS). However, this may be accounted for by the fact that discomfort in bed is likely to lead to poor sleep which in turn causes tiredness and fatigue. Evidence suggests that this is associated with adverse mental health [14].

\section{Discussion}

ALS/MND has substantial consequences in terms of impairment and disability. Individuals with the disorder may experience wide-ranging adverse effects to their quality of life [9]. To date, systematic attempts to assess ALS/MND from the perspective of the patient have been relatively limited, and have utilised generic measures or aspects of generic measures seen to be appropriate to this patient group. However, there are a number of aspects of the disease that are not addressed by generic instruments such as the SIP and SF-36. Problems such as embarrassment, selfconsciousness, difficulties of communication and abnormal bodily symptoms or discomfort that are rarely addressed by existing generic instruments can have a major impact on patients with ALS/MND. Furthermore, such measures can be long and may not seem appropriate to patients with specific diseases. The 40-item questionnaire developed in this study has been shown to be highly reliable both in terms of internal reliability at the level of the patient group and, given the magnitude of the reliability results, potentially the individual [15]. More research is of course needed to determine the applicability of this measure to individual patients in clinical care.

Content validity has been addressed by developing items on the basis of in-depth interviews with patients rather than relying on the literature of clinical scales in this field. The content of the ALSAQ-40 addresses experiences of great importance to individuals with ALS/ MND such as fear of falling when walking, difficulties cutting and eating food, participating in conversations, feelings of isolation, social embarrassment, as well as measuring feelings of fear and hopelessness about the future. Such experiences are quite distinctively associated with ALS/MND disease.

The ALSAQ-40 has construct validity in that scale scores are significantly associated (as predicted) with the scales of SF-36 that measure related experiences. Furthermore, it reflects clinicians' assessments of health status on a number of related dimensions of the ALS-FRS. The questionnaire was developed in order to be sufficiently short and simple in format so that it would be applicable to a wide range of healthcare applications.

A limited range of pharmacological therapies specifically for patients with ALS/MND is currently available [6]. However, new drugs are in development and must be assessed in terms of their impact on the well-being and functioning of patients. The role of ALSAQ-40 could be central to such assessments. The development of appropriate and feasible measures of outcome for ALS/MND is an essential step in the evaluation of such interventions [11]. This paper presents evidence of a patient-based measure for such purposes. The next stage in the assessment of the ALSAQ-40 will be its role in measuring changes over time in patients' diagnosed with ALS/MND. Copies of the questionnaire are available on request from Crispin Jenkinson.

Acknowledgements We are grateful to the Motor Neurone Disease Association, Northampton, UK, for funding this study, and to patients in both the UK and the USA for generously providing time and effort for this research.

\section{References}

1. Bensimon G, Lacomblez L, Meninger $\mathrm{V}$ and the ALS/Riluzole Study Group (1994) A controlled trial of riluzole in amyotrophic lateral sclerosis. N Engl J Med 330:585-951

2. Bernat JL, Vincent FM (1987) Neurology. Oradell, NJ: Medical Economics Books

3. Cronbach LJ (1951) Coefficient alpha and the internal structure of tests. Psychometrika 16:297-334

4. Damiano A, Patrick DL, Guzman G, et al (1999) Measurement of health related quality of life in patients with Amyotrophic Lateral Sclerosis in clinical trials of new therapies. Med Care $37: 15-26$
5. Donaghy M (1996) The motor neurone diseases. In: Weatherall DJ, Ledingham JGG, Warrell DA (eds) Oxford textbook of medicine, Third Edition, Volume 3: Oxford University Press, Oxford, pp 4087-4090

6. Lacomblez L, Bensimon G, Leigh PN, et al for the Amyotrophic Lateral Sclerosis/Riluzole Study Group II (1996) Dose-ranging study of riluzole in amyotrophic lateral sclerosis. Lancet 347 : 1425-1431

7. Leigh PN, Swash M (1995) Motor neurone disease: Biology and management. London. Springer-Verlag, London Berlin Heidelberg New York Paris Tokyo Hong Kong Barcelona Budapest
8. Nunnally JC, Bernstein IH (1994) Psychometric theory. Third edition, McGraw Hill New York

9. Robinson I, Hunter M (1998) Motor neurone disease. Routledge, London

10. Shields RK, Ruhland JL, Ross MA, et al (1998) Analysis of health-related quality of life and muscle impairment in individuals with amyotrophic lateral sclerosis using the medical outcome survey and the Tufts Quantitative Neuromuscular Exam. Arch Phys Med Rehabil $97: 855-862$

11. Swash M (1997) Health outcome and quality-of-life measurements in amyotrophic lateral sclerosis. J Neurol 244 [Suppl 2]:S26-S29 
12. Tandan R, Bradley WG (1985) Amyotrophic lateral sclerosis: Part 1. Clinical features, pathology, and ethical issues in management. Ann Neurol 18:

$271-280$
13. The ALS CNTF treatment study group (ACTS) phase I-II Study Group (1996) The Amyotrophic Lateral Sclerosis Functional Rating Scale. Assessment of activities of daily living in patients with amyotrophic lateral sclerosis. Arch Neurol 53:141-147

14. Walker EA, Katon WJ, Jemelka RP (1993) Psychiatric disorders and medical care utilization among people in the general population who report fatigue. J Gen Intern Med 8 : 436-440
15. Ware JE, Kosinski M, Keller SD (1994) SF-36 Physical and Mental Health Summary Scales: A User's Manual. The Health Institute, New England Medical Center, Boston, Massachusetts

16. Ware JE Jr, Sherbourne CD (1992)

The MOS 36-item short-form health survey (sf-36) I: Conceptual framework and item selection. Med Care 30: 473-483 